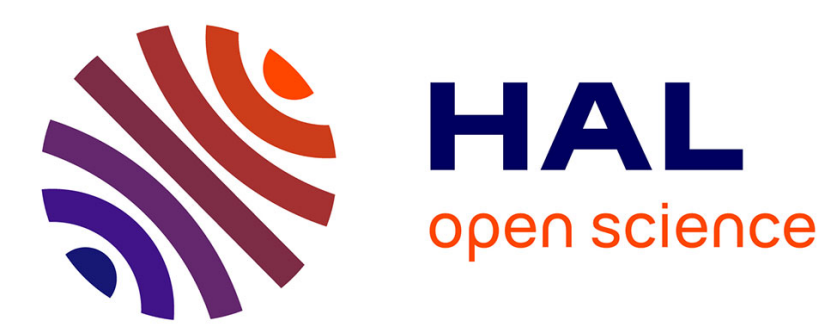

\title{
Systematic investigation of the mechanical properties of pure silica zeolites: stiffness, anisotropy, and negative linear compressibility
}

François-Xavier Coudert

\section{- To cite this version:}

François-Xavier Coudert. Systematic investigation of the mechanical properties of pure silica zeolites: stiffness, anisotropy, and negative linear compressibility. Physical Chemistry Chemical Physics, 2013, 15 (38), pp.16012. 10.1039/C3CP51817E . hal-02116883

\section{HAL Id: hal-02116883 \\ https://hal.science/hal-02116883}

Submitted on 1 May 2019

HAL is a multi-disciplinary open access archive for the deposit and dissemination of scientific research documents, whether they are published or not. The documents may come from teaching and research institutions in France or abroad, or from public or private research centers.
L'archive ouverte pluridisciplinaire HAL, est destinée au dépôt et à la diffusion de documents scientifiques de niveau recherche, publiés ou non, émanant des établissements d'enseignement et de recherche français ou étrangers, des laboratoires publics ou privés. 


\title{
Systematic Investigation of the Mechanical Properties of Pure Silica Zeolites: Stiffness, Anisotropy, and Negative Linear Compressibility ${ }^{\dagger}$
}

\author{
François-Xavier Coudert $* a$
}

While the energetic stability of the large number of possible $\mathrm{SiO}_{2}$ polymorphs has been widely addressed by both experimental and theoretical studies, there is a real dearth of information on their mechanical properties. We performed a systematic study of the elastic properties of 121 pure silica zeolites, including both experimentally synthesized and hypothetical structures, by means of density-functional theory calculations. We found that most frameworks exhibit high elastic anisotropy, and the experimentally synthesized structures are among the most mechanically stable ones. We propose to extend the "feasibility" criterion proposed in earlier literature to include elastic anisotropy, allowing to further reduce the number of possible targets for synthesis of $\mathrm{SiO}_{2}$ polymorphs. We also predict that a small number of hypothetical pure silica zeolites present large negative linear compressibility (NLC), which we attribute to the wine-rack motif of their framework.

\section{Introduction}

Pure silica zeolites are metastable porous phases of $\mathrm{SiO}_{2},{ }^{1,2}$ also known as all-silica zeolites, siliceous zeolites, zeosils, etc. Their porous structure is formed upon crystallization in the presence of templating agents, and retained after a calcination process which removes the template but does not break the structural connectivity. In addition to the traditional applications of microporous materials, such as adsorption, separation and gas storage, they have a practical application that finds its root in the hydrophobicity of their pore surface: the combination of small pore size and nonwetting surface can be leveraged to adsorb, store, or dissipate energy. ${ }^{3}$ There is also a growing enthusiasm for their use as thin films as low $k$ materials, chemical sensors, membrane reactors, and microelectronic devices. ${ }^{4-6}$

While more than 200 zeolite frameworks have been observed in natural or synthesized zeolitic materials, less than a quarter of that number (46 out of 206) can be experimentally obtained as pure silica compounds. The synthesis of new pure silica zeolites remains a considerable challenge. In order to evaluate the viability of the zeolite frameworks in their $\mathrm{SiO}_{2}$ form, researchers have worked on characterizing the lattice energies of various pure silica zeolite structures and linking it to their structural features. In their seminal work in 1994, Gale et al. ${ }^{7}$ used force field-based energy minimizations of

† Electronic Supplementary Information (ESI) available: Energies of the 163 pure silica zeolites whose geometry was optimized. Full characterization of the 121 pure silica zeolites whose elastic constants were calculated.

a CNRS \& Chimie ParisTech, UMR 7575 LECIME, 11 rue Pierre et Marie Curie, 75005 Paris, France; E-mail: fx.coudert@chimie-paristech.fr
10 silica frameworks, including 6 zeolite structures, and compared the energies obtained to that of the thermodynamically stable $\mathrm{SiO}_{2}$ polymorph $\alpha$-quartz. They linked the relative energies of the frameworks to their density, a correlation which was soon confirmed on a larger number of frameworks. ${ }^{8}$ This correlation was turned into a feasibility criterion by the observation that all known pure silica zeolites synthesized to date fall within $30 \mathrm{~kJ} / \mathrm{mol}$ (per $\mathrm{SiO}_{2}$ unit) of the dense $\alpha$-quartz phase, which was then used to assess the viability of hypothetical zeolite frameworks. ${ }^{9}$ Finally, the growth of computational power available has made it possible to perform systematic studies of the energetics of pure silica zeolite frameworks. An example of such is the recent systematic work by Sastre et al. ${ }^{10}$ based on well-established force fields, which showed that a combination of both lattice energy and density may be adopted as empirical criteria to assess the thermodynamic feasibility of the frameworks.

However, there has been no systematic exploration of the mechanical properties of pure silica zeolites to date. The elastic properties of a few specific zeolites have been explored, either experimentally ${ }^{11-13}$ or by means of molecular simulation. ${ }^{2,14-16}$ The only large-scale study of pure silica zeolites' elastic properties was performed in 2000 by Grima et al. ${ }^{14}$, based on force field energy minimization, but it was exclusively focused on materials with negative Poisson's ratio (also called auxetic materials). Other properties, such as the zeolites' Young's and shear moduli, as well as their linear compressibility, were not discussed. Nonetheless, mechanical properties are of great importance for practical applications of these microporous materials, and in particular their Young's and shear moduli. There is currently a dire need for better 
understanding how widely they can vary in a single family of materials $\left(\mathrm{SiO}_{2}\right.$ polymorphs) and how they are linked to characteristic features of the inorganic framework. This need is rendered even more pressing by the recent development of new families of metal-organic frameworks, such as the Zeolitic Imidazolate Frameworks, ${ }^{17}$ that present a large number of polymorphic crystalline structures ${ }^{18,19}$ isomorphic to zeolites, and for which the links between framework topology, linker functionalization, and energetic and mechanical properties have only recently been addressed. ${ }^{20,21}$

In the present paper, we report a large-scale study of the energetic and mechanical properties of synthesized and hypothetical pure silica zeolites. We performed quantum chemistry calculations at the density functional theory (DFT) level of theory, determining the relative energies of 163 different pure silica frameworks and the second-order elastic constants tensors of 121 of these frameworks. State-of-the-art DFT methods was shown on a series of pure silica structures to give good agreement with other methodologies and experimental data. ${ }^{22}$ In such a large scale study, the use of parameter-free DFTbased calculations avoids a dependence of the results obtained on force field calibration, which may not be able to reproduce equally well all the different zeolite frameworks (especially those with different secondary building units).

\section{Methodology}

\subsection{Energy calculations}

We started from the full list of 206 structures (at the current date) of the Database of Zeolite Structures ${ }^{23}$ maintained by the Structure Commission of the International Zeolite Association (IZA). These structures (atom coordinates and unit cell parameters) were optimized with DLS- $76^{24}$ assuming a pure $\mathrm{SiO}_{2}$ composition. These initial structures were then fully relaxed by optimizing both atomic positions and unit cell parameters. 163 pure silica structures reached convergence within the limits of the computational available: this represents $79 \%$ of all the IZA-listed frameworks. The full list of reported in Figure 1. The same calculations were also performed on the $\alpha$-quartz structure, for the purpose of comparison. All first principles calculations were performed in the density functional theory approach with periodic unit cell, full use of the crystals' symmetry elements and localized basis sets as implemented in the CRYSTAL09 code. ${ }^{25}$ We used the B3LYP hybrid exchange-correlation functional, ${ }^{26}$ with empirical correction for the dispersive interactions following the "D2" scheme of Grimme. ${ }^{27}$ All electron basis sets were used for oxygen and silicon. ${ }^{28}$ The full results (optimized energies and structures in CIF format) are available as part of the Supporting Information.

\subsection{Elastic tensor calculations}

The tensorial Hooke's law establishes a general relationship between the strain $\varepsilon$ and the stress $\sigma$ in a solid in the linear elastic regime, through the fourth-order tensor $C$ of secondorder elastic constants: ${ }^{29} \sigma_{i j}=\sum_{k l} C_{i j k l} \varepsilon_{k l}$. Taking advantage of the symmetries of stress and strain matrices and using the Voigt notation, the elasticity tensor (or stiffness tensor) $C_{i j k l}$ can be expressed as a $6 \times 6$ symmetric matrix of 21 elastic constants $C_{i j}$. The crystal system of the material considered yields additional symmetry constraints, further reducing the number of independent elastic constants: 4 for cubic crystals, 5 in the hexagonal case, 6 or 7 for the tetragonal classes, 9 for orthorhombic crystals and 13 for monoclinic crystals. ${ }^{29}$

The calculation of second-order elastic constants of the materials studied were performed with the use of the CRYSTAL09 code, ${ }^{25}$ whose implementation was detailed in Ref. 30. In short, starting from the optimized geometry of the relaxed crystal, each deformation mode of the crystal is scanned and the second-order elastic constants can be calculated by finite differences as second derivatives of the energy:

$$
C_{i j}=\frac{1}{V}\left(\frac{\partial^{2} E}{\partial \varepsilon_{i} \partial \varepsilon_{j}}\right)
$$

For each deformation mode, 5 different values of strain were sampled (including the relaxed structure), with a total amplitude of $\pm 2 \%$. The accuracy of this methodology is now well established for the calculation of elastic constants in dense inorganic crystals ${ }^{30}$ as well as porous materials: carbon nanotubes, ${ }^{31}$ boron nitride nanotubes ${ }^{32}$ and metal-organic frameworks. ${ }^{33-35}$

From the second-order elastic constants of each material, a full tensorial analysis was performed ${ }^{35,36}$ (see ref. 35 for details) and key quantities were derived that characterize the mechanical behavior of the structure in the elastic regime: Young's modulus, shear modulus and linear compressibility. In an anisotropic medium such as a crystal, these properties are directional; for each property, we calculated the minimal and maximal values reached.

These calculations of elastic tensor were performed on a total of 121 pure silica zeolite structures, i.e. $59 \%$ of the IZA database (see Figure 1). These included 13 cubic structures, 26 hexagonal structures, 21 tetragonal structures, 40 orthorhombic structures and 21 monoclinic structures. The full results are available as a spreadsheet as part of the Supporting Information.

\section{Results and discussion}

\subsection{Energetics of pure silica zeolites}

Following the methodology of earlier works ${ }^{7,10}$ for studying the feasibility of pure silica zeolites, we show in Figure 2 a 
plot of lattice energy relative to $\alpha$-quartz versus specific volume, for all 163 frameworks whose structure was optimized in this work. Overall, an inverse correlation between stability and specific volume is found, as is expected (i.e. lattice energy increases with volume, or decreases with density). We also see, despite this overall correlation, there is significant spread of the energies: in addition to the RWY framework, an outlier nearly $100 \mathrm{~kJ} / \mathrm{mol}$ less stable than $\alpha$-quartz, a large number of structures are very high in energy. The list of frameworks with $\Delta E>30 \mathrm{~kJ} / \mathrm{mol}$ include OSO, JST, OBW, BOZ, NPT, SOS, BSV, CZP, WEI, PUN and AFY. Moreover, we can see that frameworks types that have been experimentally synthesized as pure silica zeolites (red symbols in Fig. 2) all have relatively low lattice energy, the larger one being FAU at $\Delta E \approx 22 \mathrm{~kJ} / \mathrm{mol}$. The left pane of Figure 2 shows a zoom on this low-energy region of the graph. There, it can be seen that all known pure silica frameworks fall in a gray band of width $\pm 15 \mathrm{~kJ} / \mathrm{mol}$. In addition, a large number of hypothetical pure silica materials also fall in that region of the energy-density diagram, indicating that with the right choice of template and synthesis conditions, their synthesis should be achievable. Of particular interest on this diagram are the points of lowest energy at a given density, such as MEP, SFN, UFI and VFI.

In addition, we can use these results to find correlations between lattice energy and other properties of the zeolite frameworks. We start by noting that, among the synthesized pure silica zeolites, those with $0 \mathrm{D}$ and $1 \mathrm{D}$ pore networks are present in abundant numbers, while those with three-dimensional channel system are underrepresented (see Table 1). In order to understand this effect, we plot in Figure 3 the histograms of lattice energies for channels as a function of the dimensionality of their pore network. There is an overall increase of lattice energy from $0 \mathrm{D}$ to $3 \mathrm{D}$, which can be attributed to the energy-density correlation: networks with a channel system of higher dimension are typically less dense. Furthermore, we note that all the pure silica zeolites with a lattice energy higher than $30 \mathrm{~kJ} / \mathrm{mol}$ per $\mathrm{SiO}_{2}$ have 3D channels, with the exception of the ASV framework.

These findings are overall in good agreement with the earlier study by Bushuev et al., ${ }^{10}$ therefore confirming the conclusions of their work and validating our DFT-based approach. We refer the reader to the discussion in ref. 10 for a longer discussion of the structure/stability relationship in pure silica zeolites, and focus in the rest of this manuscript on their mechanical properties.

\subsection{Young's and shear moduli}

We calculated the second-order elastic constants of 121 pure silica zeolite structures, of which 92 are hypothetical structures and 29 have been experimentally synthesized. Among the various elastic properties we calculated from those elas- tic tensors, we focus in this section on the Young's and shear moduli, which characterizes the stiffness of the material in response to uniaxial compression and shear, respectively. Because of the anisotropic nature of the crystals, these elastic moduli are directional, i.e. they vary in space with the direction of the applied stress. Because one of the main interest in mechanical properties of zeolites is their usually high mechanical stability, we discuss here the minimum Young's and shear moduli for each framework; their anisotropy and its impact will be discussed in the next section.

Figure 4 provides a graphical representation of the 121 pure silica zeolites' mechanical properties in a Young's modulusshear modulus space. We first note the very impressive range of both moduli in this family of materials, despite all of them being formed of the same strong $\mathrm{Si}-\mathrm{O}$ bonds: the minimal Young's modulus for pure silica zeolites ranges from $11.5 \mathrm{GPa}$ (SOS) to $114.5 \mathrm{GPa}$ (MTN), and the minimal shear modulus from $3.7 \mathrm{GPa}$ (OWE) to $42.2 \mathrm{GPa}(\mathrm{MTN})$. For each modulus, the variations span an order of magnitude, which is remarkable. We also see, as in most materials, shear modulus is usually weaker than Young's modulus; however, both moduli a strongly correlated. We thus propose to follow an alternative way to characterize the "softness" of the frameworks, by looking at the smallest eigenvalue $\lambda_{1}$ of the $6 \times 6$ matrix of elastic constants. This lowest eigenvalue corresponds to the softest elastic modulus, i.e. to the stiffness of the material in the softest deformation mode (the associated eigenvector), whether this deformation mode is pure compression, pure shear or sum of the two. This approach was recently proposed and validated in a study of the flexibility of metal-organic frameworks. ${ }^{35}$

Another point that can be seen from Figure 4 is that, while there is a certain spread, most of the $\mathrm{SiO}_{2}$ frameworks experimentally attested have medium to large stiffness. In fact, only 3 of the synthesized pure silica frameworks have a Young's modulus lower than $35 \mathrm{GPa}$, while it accounts for more than a third (36\%) of the hypothetical frameworks. Just like there is a known correlation between experimental feasibility and lattice energy, there seem to be a correlation between feasibility and stiffness. In order to understand this correlation better and to check whether it might be an indirect correlation via a third quantity, we plotted in Figure 5 the correlations between density, lattice energy and stiffness (as measured by the smallest eigenvalue, $\lambda_{1}$, of the elastic matrix). The results show little in the way of correlation between stiffness and density (or specific volume; left panel of Fig. 5): in that regard, mechanical stiffness is quite different from energetic feasibility, which presents a clear correlation, although with some spread. We can provide a quantitative confirmation of this conclusion by calculating Pearson's correlation coefficient for both sets of data: it is -0.21 for stiffness vs. density, compared to 0.72 for energy vs. density.

On the right panel of Fig. 5, we can check the correlation be- 
tween stiffness and lattice energy. There is now a modest correlation (Pearson's $r$ of -0.29 ), which becomes quite strong if you focus on the most feasible frameworks: Pearson's $r$ is -0.57 for the points with $\Delta E \geq 25 \mathrm{~kJ} / \mathrm{mol}$; the corresponding linear regression is plotted as dashed line in Fig. 5). This clear correlation is somewhat unexpected, as it links the relative stability of a framework with its elasticity, which is a local property. However, we find no correlation between the stiffness of the pure silica zeolites and the crystal class (symbol color in Fig. 5) or symmetry of their framework. These two facts lead us to conclude that an important factor in the variations of stiffness within the family of pure silica zeolites is the deformation of the $\mathrm{Si}-\mathrm{O}$ bond lengths and $\mathrm{Si}-\mathrm{O}-\mathrm{Si}$ angles in the frameworks: when bond lengths and bond angles are strained, the lattice energy of the framework increases and the framework stiffness diminishes, because its is weakened by the strain.

\subsection{Anisotropy of the elastic properties}

As indicated earlier, because of the anisotropic nature of crystals, monocrystalline elastic moduli are directional: Young's modulus $E(\mathbf{u})$ depends on a direction, indicated by unit vector $\mathbf{u}$, and the shear modulus $G(\mathbf{u}, \mathbf{n})$ depends on the shear direction $\mathbf{u}$ and shear plane normal $\mathbf{n}$. We investigate here the anisotropy in pure silica zeolites's elastic properties by looking at the ratio of their maximal and minimal values. Figure 6 reports the $\left(E_{\max }, E_{\min }\right)$ and $\left(G_{\max }, G_{\min }\right)$ plots of the 121 pure silica zeolites studied in this work. While some frameworks have little anisotropy (i.e. correspond to a point near the $y=x$ line in these diagrams), most pure silica zeolites exhibit a large anisotropy in both their Young's and shear moduli. Some frameworks exhibit almost an order of magnitude difference between their stiffest deformation mode and their softest one.

The extent of the anisotropy of the two elastic moduli is an important quantity: very high anisotropy usually indicates limited mechanical stability of the material, which is in turn a limiting factor for practical applications. For this reason, we performed a more quantitative analysis of elastic anisotropy, we define the anisotropy $\eta$ as:

$$
\eta=\min \left(\frac{E_{\max }}{E_{\min }}, \frac{G_{\max }}{G_{\min }}\right)
$$

We then propose to plot the experimental and hypothetical pure silica zeolites in an energy-stability diagram $(\Delta E, \eta)$. It clearly appears, on that diagram (Figure 7), that indeed mechanical stability plays some role in the experimental feasibility of zeolite frameworks as pure $\mathrm{SiO}_{2}$ materials: most of the experimentally synthesized frameworks are grouped in bottom-left area of the plot, corresponding to both low lattice energy and low elastic anisotropy. We thus propose to add mechanical stability, as characterized by low anisotropy of the elastic properties, as a new criterion to feasibility of pure silica frameworks (in addition to the established density and energy criteria). We suggest that materials satisfying the conditions of $\Delta E \leq 20 \mathrm{~kJ} / \mathrm{mol}$ per $\mathrm{SiO}_{2}$ and $\eta \leq 4$ may be the most promising pure silica frameworks yet undiscovered. From our calculations, the list of most feasible framework types would include MEP, CAS, LOS, LIO, AFG, ATO, CAN, EPI, DAC and BCT.

\subsection{Zeolites with negative linear compressibility}

Negative linear compressibility (NLC) is a counterintuitive property of some materials which, under hydrostatic compression, see one or more of their linear dimensions decrease. Thermodynamics imposes that the bulk compressibility of a material, which is the inverse of its bulk modulus, be positive: isotropic compression must lead to a decrease in volume. For most materials, this decrease in volume stems from a decrease in all of their linear dimensions. However, a handful of materials show NLC, i.e. while their overall volume diminishes upon compression, one or more of their dimensions actually increase in the process. NLC is a rare and highly desirable property, and practical applications of materials exhibiting NLC include artificial muscles, actuators and pressure sensors. ${ }^{37}$ In the search for materials with the largest possible NLC, researchers have looked for structures with specific structural motifs, and in particular the wine-rack ${ }^{38}$ and honeycomb ${ }^{37}$ topologies. Recent reports have evidenced NLC in metal-organic frameworks, ${ }^{39}$ including several cases of colossal negative linear compressibility in compliant metal-organic frameworks based on the wine-rack motif. ${ }^{34,35}$ However, to our knowledge, NLC has never been demonstrated in a zeolite yet.

16 of the 121 pure silica zeolites studied in this work exhibit negative linear compressibility, which corresponds to $13 \%$ of the frameworks studied. We thus predict that NLC is not a rare phenomenon in zeolites, though it has never been demonstrated experimentally, nor hypothesized from $a b$ initio calculations. The extent of the NLC of pure silica zeolites, measured by the linear compressibility $\beta_{\min }$ in the direction where it is the most negative, varies between $\beta_{\min }=-0.2 \mathrm{TPa}^{-1}$ (for the AFN framework) and $\beta_{\text {min }}=$ $-13.7 \mathrm{TPa}^{-1}$ (for the GIS framework). The later is a remarkably large value, in the same range as the strongest NLC effects discovered very recently for inorganic materials $\left(-75 \mathrm{TPa}^{-1}\right.$ for $\mathrm{Ag}_{3}\left[\mathrm{Co}\left(\mathrm{CN}_{6}\right)\right] ;{ }^{40}-42 \mathrm{TPa}^{-1}$ for $\mathrm{Zn}\left[\mathrm{Au}\left(\mathrm{CN}_{2}\right)\right]_{2} ;{ }^{37}-12 \mathrm{TPa}^{-1}$ for $\left.\mathrm{KMn}\left[\mathrm{Ag}\left(\mathrm{CN}_{2}\right)\right]_{3}{ }^{41}\right)$. Moreover, GIS is among the "feasible" pure silica zeolites, with a lattice energy of $17.4 \mathrm{~kJ} / \mathrm{mol}$ per $\mathrm{SiO}_{2}$ relative to $\alpha$-quartz, and a specific volume of $0.63 \mathrm{~cm}^{3} / \mathrm{g}$. It is thus a good candidate for the synthesis of a pure silica zeolite with large negative 
linear compressibility. Other candidates are ATT, ABW, APC, MSO, MER, PHI, UEI, AHT, AWO and LAU.

Finally, looking at the frameworks that exhibit NLC (the three frameworks with the largest NLC are depicted in Fig. 8) immediately reveals that they share a common feature: they all have a wine-rack topology. Thus, while NLC in zeolites has not been reported before, its mechanism is quite similar to that observed in dense inorganic materials, molecular frameworks and porous metal-organic frameworks.

\section{Conclusion}

We have performed the first systematic study of the elastic properties of pure silica zeolites. By means of density functional theory calculations, we have calculated the secondorder elastic constants of $121 \mathrm{SiO}_{2}$ frameworks, including members that have already been synthesized experimentally as well as hypothetical structures. We showed that DFT calculations confirm the correlation established by earlier work between framework density and energetic stability. In order to shed light into the little-known mechanical behavior of pure silica zeolites, we analyzed their Young's and shear moduli, as well as their linear compressibility. We found that most of the frameworks studied have high anisotropy of their elastic properties. However, the frameworks that have been experimentally synthesized tend to be among the most mechanically stable ones, with relative low anisotropy. Thus, we propose to extend the "feasibility" criterion proposed in earlier literature for pure silica zeolites to include elastic anisotropy, allowing to further reduce the number of possible targets for synthesis of $\mathrm{SiO}_{2}$ frameworks. Finally, we predict that a small number of hypothetical pure silica zeolites present large negative linear compressibility (NLC) due the wine-rack motif of their framework. If they were synthesized, such inorganic nanoporous materials with NLC could have applications as nano-actuators, pressure or gas sensors.

\section{Acknowledgement}

The author thanks German Sastre for a helpful discussion. This work was performed using HPC resources from GENCIIDRIS (project 087069).

\section{References}

1 S. M. Auerbach, K. A. Carrado and P. K. Dutta, Handbook of Zeolite Science and Technology, CRC Press, 2003.

2 R. Astala, S. M. Auerbach and P. A. Monson, J. Phys. Chem. B, 2004, 108, 9208-9215.

3 V. Eroshenko, R.-C. Regis, M. Soulard and J. Patarin, J. Am. Chem. Soc., 2001, 123, 8129-8130.

4 M. E. Davis, Nature, 2002, 417, 813-821.
5 E. McLeary, J. Jansen and F. Kapteijn, Microporous and Mesoporous Materials, 2006, 90, 198-220.

6 J. Caro and M. Noack, Microporous and Mesoporous Materials, 2008, 115, 215-233.

7 N. J. Henson, A. K. Cheetham and J. D. Gale, Chem. Mater., 1994, 6, 1647-1650.

8 K. de Boer, A. Jansen and R. van Santen, Phys. Rev. B, 1995, 52, 12579 12590.

9 M. W. Deem, R. Pophale, P. A. Cheeseman and D. J. Earl, J. Phys. Chem. $C, 2009, \mathbf{1 1 3}, 21353-21360$.

10 Y. G. Bushuev and G. Sastre, J. Phys. Chem. C, 2010, 114, 19157-19168.

11 J. Lin, X. Shu and J. Dong, Materials Letters, 2005, 59, 1595-1597.

12 Z. Li, M. C. Johnson, M. Sun, E. T. Ryan, D. J. Earl, W. Maichen, J. I. Martin, S. Li, C. M. Lew, J. Wang, M. W. Deem, M. E. Davis and Y. Yan, Angew. Chem. Int. Ed., 2006, 45, 6329-6332.

13 C. Sanchez-Valle, S. V. Sinogeikin, Z. A. D. Lethbridge, R. I. Walton, C. W. Smith, K. E. Evans and J. D. Bass, J. Appl. Phys., 2005, 98, 053508.

14 J. N. Grima, R. Jackson, A. Alderson and K. E. Evans, Adv. Mater, 2000, 12, 1912-1918.

15 J. N. Grima, R. Gatt, V. Zammit, J. J. Williams, K. E. Evans, A. Alderson and R. I. Walton, J. Appl. Phys., 2007, 101, 086102.

16 J. J. Williams, K. E. Evans and R. I. Walton, Appl. Phys. Lett., 2006, 88, 021914.

17 K. S. Park, Z. Ni, A. P. Côté, J. Y. Choi, R. Huang, F. J. Uribe-Romo, H. K. Chae, M. O'Keeffe and O. M. Yaghi, P. Natl. Acad. Sci., 2006, 103, 10186-10191.

18 I. A. Baburin, S. Leoni and G. Seifert, J. Phys. Chem. B, 2008, 112, $9437-$ 9443.

19 D. W. Lewis, A. R. Ruiz-Salvador, A. Gómez, L. M. Rodriguez-Albelo, F.-X. Coudert, B. Slater, A. K. Cheetham and C. Mellot-Draznieks, CrystEngComm, 2009, 11, 2272.

20 J. C. Tan and A. K. Cheetham, Chem. Soc. Rev., 2011, 40, 1059.

21 A. U. Ortiz, A. Boutin, A. H. Fuchs and F.-X. Coudert, J. Phys. Chem. Lett., 2013, 4, 1861-1865.

22 M. A. Zwijnenburg, F. Corá and R. G. Bell, J. Phys. Chem. B, 2007, 111, 6156-6160.

23 Database of Zeolite Structures, Structure Commission of the International Zeolite Association, http://www.iza-structure.org/databases/.

24 Ch. Baerlocher, A. Hepp, W. M. Meier, DLS-76. Distance Least Squares Refinement Program (Institut für Kristallographie, ETH Zürich, 1977).

25 R. Dovesi, R. Orlando, B. Civalleri, C. Roetti, V. R. Saunders and C. M. Zicovich-Wilson, Z. Kristallogr., 2005, 220, 571-573.

26 A. D. Becke, J. Chem. Phys., 1993, 98, 5648.

27 S. Grimme, J. Comput. Chem., 2006, 27, 1787.

28 R. Nada, J. B. Nicholas, M. I. McCarthy and A. C. Hess, Int. J. Quantum Chem., 1996, 60, 809-820.

29 J. F. Nye, Physical Properties of Crystals - Their Representation by Tensors and Matrices, Clarendon, 1985.

30 W. Perger, J. Criswell, B. Civalleri and R. Dovesi, Comput. Phys. Commun., 2009, 180, 1753-1759.

31 R. Demichelis, Y. Noël, P. D’Arco, M. Rérat, C. M. Zicovich-Wilson and R. Dovesi, J. Phys. Chem. C, 2011, 115, 8876-8885.

32 A. Erba, M. Ferrabone, J. Baima, R. Orlando, M. Rérat and R. Dovesi, J. Chem. Phys., 2013, 138, 054906.

33 J.-C. Tan, B. Civalleri, C.-C. Lin, L. Valenzano, R. Galvelis, P.-F. Chen, T. Bennett, C. Mellot-Draznieks, C. Zicovich-Wilson and A. Cheetham, Phys. Rev. Lett., 2012, 108, 095502.

34 A. U. Ortiz, A. Boutin, A. H. Fuchs and F.-X. Coudert, Phys. Rev. Lett., 2012, 109, 195502.

35 A. U. Ortiz, A. Boutin, A. H. Fuchs and F.-X. Coudert, J. Chem. Phys, 2013, 138, 174703.

36 A. Marmier, Z. A. D. Lethbridge, R. I. Walton, C. W. Smith, S. C. Parker 


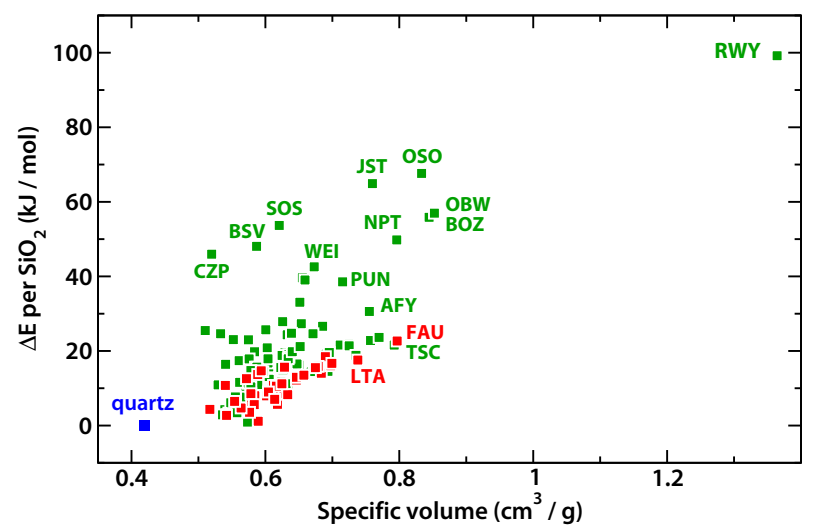

\begin{tabular}{ccccc}
\hline & $0 \mathrm{D}$ & $1 \mathrm{D}$ & $2 \mathrm{D}$ & $3 \mathrm{D}$ \\
synthesized & $7(18 \%)$ & $15(38 \%)$ & $8(21 \%)$ & $9(23 \%)$ \\
total & $16(10 \%)$ & $52(32 \%)$ & $34(21 \%)$ & $61(37 \%)$ \\
\hline
\end{tabular}

Table 1 Distribution of the pure silica zeolites with respect to the dimensionality of their framework.

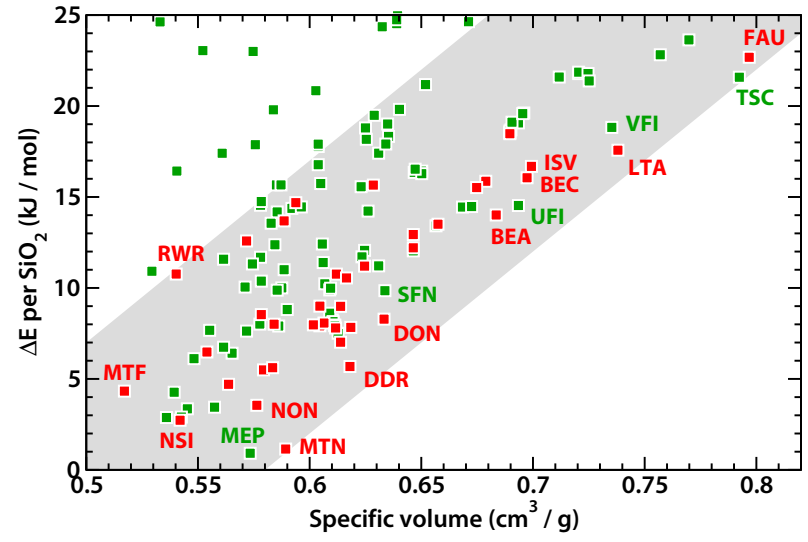

Fig. 2 Energy of the pure silica zeolites relative to $\alpha$-quartz versus specific volume. Left: all material studied; right: zoom on the low energy part. Red symbols: materials experimentally synthesized; green symbols: other topologies; blue: $\alpha$-quartz, the reference material.

and K. E. Evans, Comput. Phys. Commun., 2010, 181, 2102-2115.

37 A. B. Cairns, J. Catafesta, C. Levelut, J. Rouquette, A. van der Lee, L. Peters, A. L. Thompson, V. Dmitriev, J. Haines and A. L. Goodwin, Nat Mater, 2013, 12, 212-216.

38 A. D. Fortes, E. Suard and K. S. Knight, Science, 2011, 331, 742-746.

39 W. Li, M. R. Probert, M. Kosa, T. D. Bennett, A. Thirumurugan, R. P. Burwood, M. Parinello, J. A. K. Howard and A. K. Cheetham, J. Am. Chem. Soc., 2012, 134, 11940-11943.

40 A. L. Goodwin, D. A. Keen and M. G. Tucker, Proc. Nat. Acad. Sci. USA, 2008, 105, 18708-18713.

41 A. B. Cairns, A. L. Thompson, M. G. Tucker, J. Haines and A. L. Goodwin, J. Am. Chem. Soc., 2012, 134, 4454-4456.

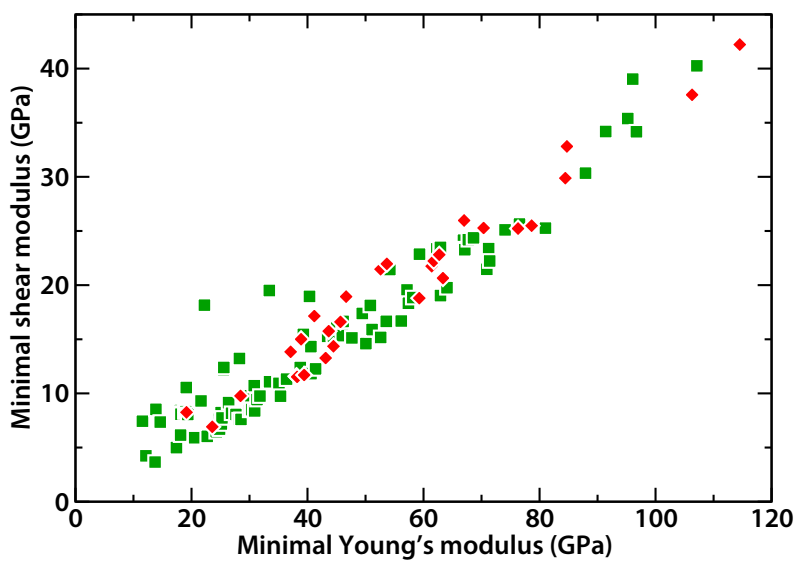

Fig. 4 Young's modulus and shear modulus of pure silica zeolites: each point represent a single framework, red points correspond to synthesized pure silica zeolites. 


\begin{tabular}{|c|c|c|c|c|c|c|c|c|c|c|c|}
\hline ABW & $\mathrm{ACO}$ & AEI & AEL & AEN & AET & AFG & AFI & AFN & AFO & AFR & AFS \\
\hline AFT & AFX & AFY & АНT & ANA & APC & APD & AST & ASV & ATN & ATO & ATS \\
\hline ATT & ATV & AWO & AWW & BCT & *BEA & BEC & BIK & BOF & BOG & BOZ & $\mathrm{BPH}$ \\
\hline BRE & BSV & CAN & CAS & CDO & CFI & CGF & CGS & CHA & $-\mathrm{CHI}$ & -CLO & CON \\
\hline CZP & DAC & DDR & DFO & DFT & DOH & DON & EAB & EDI & EMT & EON & EPI \\
\hline ERI & ESV & ETR & EUO & EZT & FAR & FAU & FER & FRA & GIS & GIU & GME \\
\hline GON & GOO & HEU & IFR & IHW & IMF & IRR & ISV & ITE & ITH & ITR & -ITV \\
\hline ITW & IWR & IWS & IWV & IWW & JBW & $\mathrm{JOZ}$ & JRY & JSN & JST & JSW & KFI \\
\hline LAU & LEV & LIO & -LIT & LOS & LOV & LTA & LTF & LTJ & LTL & LTN & MAR \\
\hline MAZ & MEI & MEL & MEP & MER & MFI & MFS & MON & MOR & MOZ & *MRE & MSE \\
\hline MSO & MTF & MTN & MTT & MTW & MVY & MWW & NAB & NAT & NES & NON & NPO \\
\hline NPT & NSI & OBW & OFF & OSI & oso & OWE & -PAR & PAU & PCR & PHI & PON \\
\hline PUN & $\mathrm{RHO}$ & -RON & RRO & RSN & RTE & RTH & RUT & RWR & RWY & SAF & SAO \\
\hline SAS & SAT & SAV & SBE & SBN & SBS & SBT & SFE & SFF & SFG & SFH & SFN \\
\hline SFO & SFS & ${ }^{*}$ SFV & SGT & SIV & SOD & SOF & sos & SSF & SSY & STF & STI \\
\hline *STO & STT & STW & -SVR & SZR & TER & THO & TOL & TON & TSC & TUN & UEI \\
\hline UFI & UOS & UOZ & USI & UTL & UWY & VET & VFI & VNI & VSV & WEI & -WEN \\
\hline YUG & ZON & & & & & & & & & & \\
\hline
\end{tabular}

Fig. 1 List of the 206 zeolite frameworks of the IZA database. In red: pure silica zeolites that have been synthesized to date; with blue background: energy optimization and elastic constants calculated in this work; green background: energy optimization performed in this work.
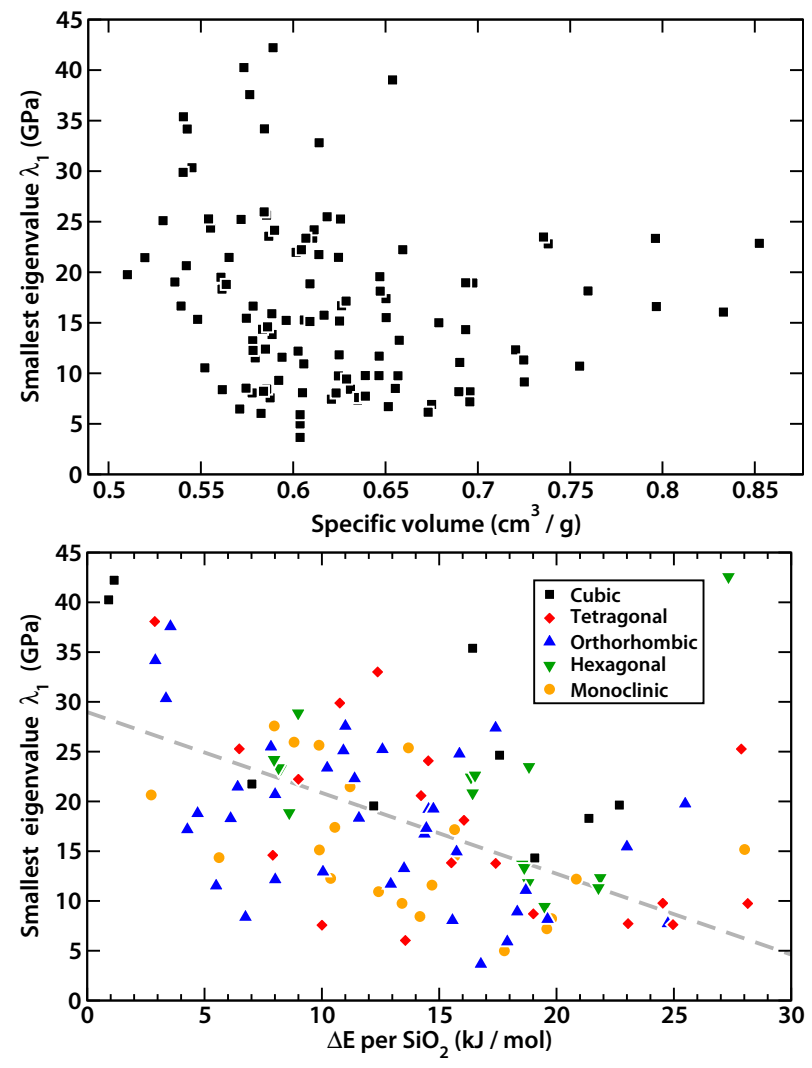

Fig. 5 Left: correlation between stiffness (measured by smallest eigenvalue of the elastic matrix, $\left.\lambda_{1}\right)$ and specific volume. Right: correlation between stiffness and lattice energy relative to $\alpha$-quartz; different colors correspond to the different lattice systems.

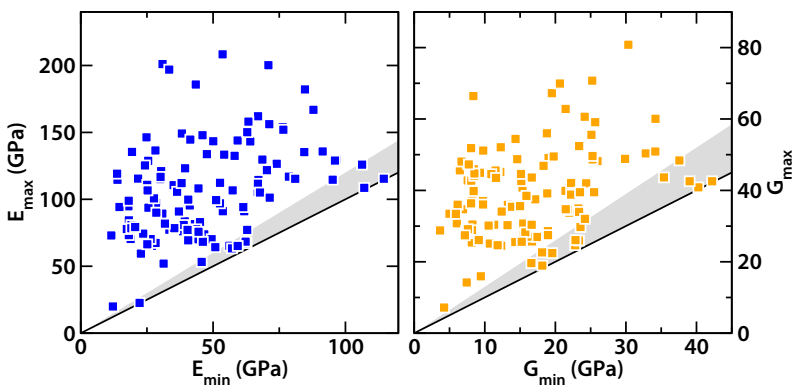

Fig. 6 Anisotropy of the Young's modulus ( $E$, left) and shear modulus $(G$, right) of pure silica zeolites, by plotting their minimal value against their maximal value. The black lines are the $y=x$ axes, and the grayed areas indicates anisotropy lower than $20 \%$. 


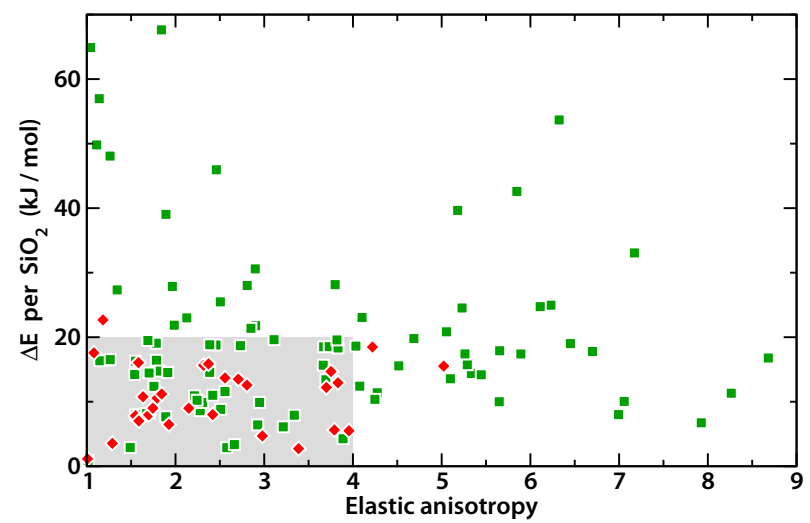

Fig. 7 Plot of the elastic anisotropy of pure silica zeolites vs. lattice energy relative to $\alpha$-quartz; red points correspond to synthesized pure silica zeolites. The gray area corresponds to the feasibility criterion proposed in the text. 

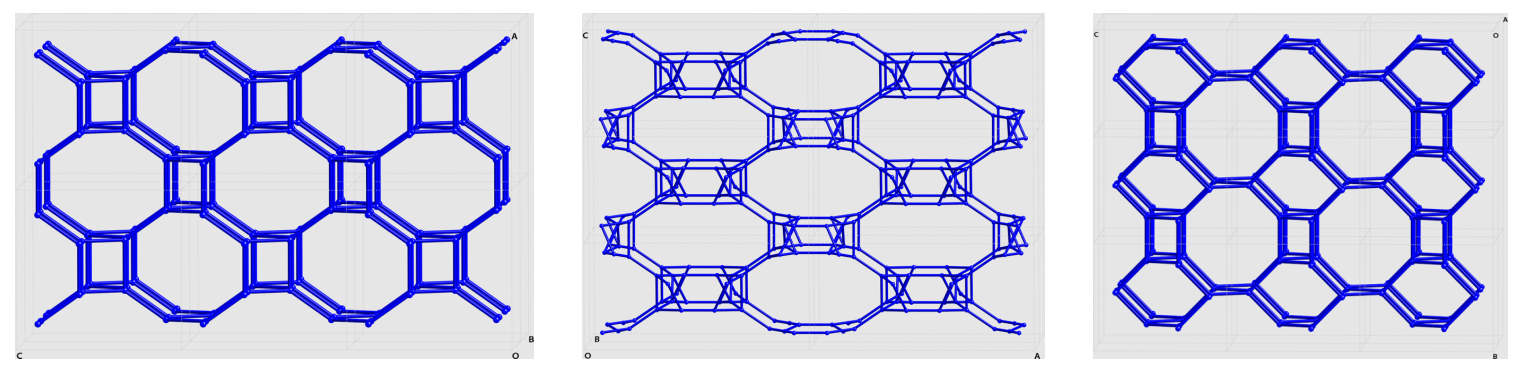

Fig. 8 Representations of the GIS, SOS and ATT zeolite frameworks (from left to right). Edges correspond to $\mathrm{Si}$ atoms, vertices to $\mathrm{Si}-\mathrm{O}-\mathrm{Si}$ links. 